\title{
Práticas de autocuidado desenvolvidas por gestantes atendidas em um ambulatório de pré-natal
}

\author{
Self-care practices developed by pregnant women in a prenatal outpatient clinic
}

Prácticas de autocuidado desarrolladas por gestantes atendidas en ambulatorio de prenatal

Sueli Riul da Silva ${ }^{1}$, Bibiane Dias Miranda Parreira ${ }^{2}$, Flávia Aparecida Dias ${ }^{3}$, Larissa Emmanuele Cardoso ${ }^{4}$, Jéssica Daher da Cunha ${ }^{5}$

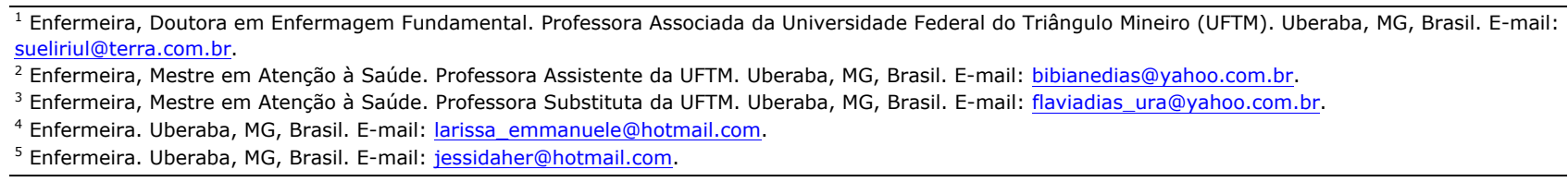

\section{RESUMO}

Trata-se de um estudo descritivo, transversal e quantitativo cujo objetivo foi verificar práticas de autocuidado desenvolvidas por gestantes atendidas pelo serviço de pré-natal de um ambulatório. Participaram 99 gestantes. A pesquisa foi realizada em um ambulatório público de um município do interior de Minas Gerais. Utilizou-se questionário. As respostas foram analisadas por estatística descritiva. Os resultados evidenciaram maior autocuidado em relação ao consumo de substâncias tóxicas, como álcool e drogas, higiene, repouso e alimentação. Cuidados, como exercícios físicos, protetor solar e cuidados com as mamas, não foram considerados como prioridades pelas gestantes. A maioria afirmou ter recebido orientações sobre autocuidado de profissionais de saúde, destacando-se o médico e enfermeiro. A problematização do tema pode contribuir para a reorganização dos serviços de saúde, a fim de garantir melhores estratégias na qualidade da atenção prestada às gestantes, em especial no desenvolvimento das práticas educativas.

Descritores: Autocuidado; Cuidado Pré-Natal; Gravidez; Educação em Enfermagem.

\section{ABSTRACT}

The objective of this descriptive, cross-sectional, quantitative study was to examine self-care practices developed by pregnant women in a prenatal outpatient clinic. Ninety-nine pregnant women participated. The survey was conducted in a public outpatient clinic in Minas Gerais. A questionnaire was administered. The responses were analyzed using descriptive statistics. The results indicated greater self-care in relation to consumption of toxic substances (alcohol and drugs), hygiene, rest and nutrition. Others, such as physical exercise, wearing sunscreen and breast care were not deemed as priorities by the participants. Most reported receiving self-care guidance from health professionals, especially physicians and nurses. Defining the nature of the theme could contribute to the reorganization of health services, in order to provide better strategies for delivering quality care to pregnant women, especially the development of educational practices.

Descriptors: Self Care; Prenatal Care; Pregnancy; Education, Nursing.

\section{RESUMEN}

Estudio descriptivo, transversal y cuantitativo que objetivó verificar las prácticas de autocuidado desarrolladas por gestantes atendidas por el servicio prenatal de un ambulatorio. Participaron 99 gestantes. La investigación fue realizada en un Ambulatorio público de un municipio del interior de Minas Gerais. Se utilizó un cuestionario. Las respuestas fueron analizadas mediante estadística descriptiva. Los resultados evidenciaron mayor autocuidado en relación al consumo de substancias tóxicas como alcohol y drogas, higiene, reposo y alimentación. Otros cuidados, como ejercicios físicos, uso de protector solar y cuidados con las mamas no fueron considerados como prioritarios por las gestantes. La mayoría afirmó haber recibido información sobre autocuidado de los profesionales de salud, destacando a médicos y enfermeros. La problematización del tema puede ayudar en la reorganización de los servicios de salud, a efectos de garantizar mejores estrategias en la calidad de la atención brindada a las gestantes, particularmente, en el desarrollo de prácticas educativas.

Descriptores: Autocuidado; Atención Prenatal; Embarazo; Educación en Enfermería. 


\section{INTRODUÇÃO}

A gestação proporciona as mulheres vivenciar experiências singulares decorrentes de modificações fisiológicas e psicossociais, fato esse que leva a necessidade de cuidados especiais. A equipe de saúde deve acolher e desenvolver cuidados com o objetivo de prevenir riscos e promover uma gravidez saudável. As políticas de saúde da mulher proporcionam a garantia do cuidado integral, sendo estas fundamentadas e implementadas pelas redes assistenciais de saúde ${ }^{(1)}$.

O acompanhamento pré-natal tem como objetivo assegurar o desenvolvimento da gestação, permitindo o parto de um recém-nascido saudável, sem impacto para a saúde materna, inclusive abordando aspectos psicossociais e as atividades educativas e preventivas ${ }^{(2)}$. É de fundamental importância para diminuir os riscos de mortalidade materna e perinatal. Talvez o principal indicador do prognóstico ao nascimento seja o acesso à assistência pré-natal. Este torna-se portanto, um momento privilegiado para discutir e esclarecer questões que são únicas para cada mulher e seu parceiro, aparecendo de forma individualizada. Temas que são tabus poderão suscitar dúvidas ou a necessidade de esclarecimentos $^{(2)}$.

Uma revisão integrativa sobre a experiência de mulheres no cuidado pré-natal destacou que a educação em saúde é fundamental para as gestantes. As mulheres valorizam as informações adquiridas, assim como a oportunidade de conversar informalmente sobre preocupações presentes neste momento ${ }^{(3)}$.

Em um estudo quase-experimental realizado no Irã, com 90 gestantes atendidas em um centro de saúde, os autores identificaram que após a divisão em dois grupos de intervenções educativas diferentes e outro grupo controle, as atividades de orientação levaram à mudança na pontuação de conhecimento sobre saúde materna, saúde bucal materna entre outras, em comparação com o grupo que não teve orientação. O que ressalta a importância das práticas educativas como forma de aquisição de novos conhecimentos e reforço positivos dos saberes já existentes ${ }^{(4)}$.

Ações de educação em saúde durante o pré-natal possibilitam a troca de informações e experiências entre mulheres e profissionais de saúde. As atividades educativas devem integrar companheiros e familiares para que questões de saúde sexual e reprodutiva sejam compartilhadas e debatidas em família, gerando um melhor entendimento quanto ao processo gestacional e os cuidados que esse exige da mulher ${ }^{(5)}$.
A assistência profissional exercida durante o prénatal pode ser o primeiro contato da gestante com os serviços de saúde, portanto deve ter qualidade técnicocientifica adequada, além de buscar atender suas necessidades. $E$ é neste momento que cabe ao profissional de saúde transparecer confiança a esta usuária e facilitar seu acesso ao serviço de saúde contribuindo para que o acompanhamento do pré-natal se mantenha até o momento do parto ${ }^{(6)}$.

Quando as intercorrências e dúvidas que a gestante apresentam são satisfeitas com orientações competentes, o resultado é o alívio e o melhor enfrentamento do período gestacional. Todo usuário do sistema de saúde tem o direito de ser orientado quanto as medidas que produzam benefícios a sua qualidade de vida, nesse sentido, uma gestante informada poderá exercer melhores cuidados com sua saúde durante a gravidez $^{(7)}$.

As pessoas têm o direito de serem informadas sobre os cuidados de saúde e participarem das decisões que influenciam suas vidas, sua saúde e os serviços comunitários. Nessa perspectiva, as chances das gestantes virem a adotar medidas de autocuidado, com vistas ao alcance de metas de saúde, tornam-se concretas $^{(7)}$. A falta de atividades educativas no pré-natal compromete o cuidado, uma vez que esta proporciona subsídios para ampliar o conhecimento e a autonomia das gestantes ${ }^{(1)}$.

Frente a esse panorama, espera-se contribuir para a melhoria das ações educativas dos enfermeiros direcionadas às gestantes nas unidades de saúde, pois, expor e satisfazer as necessidades relacionadas às intercorrências e alterações experimentadas durante a gestação, beneficia a mulher no alívio e enfrentamento $\operatorname{destas}^{(7)}$.

Na Colômbia, foi desenvolvido um estudo, no qual foram elaboradas diretrizes sobre prevenção, detecção e tratamento precoce de complicações e sintomatologias relacionadas à gravidez a ser adotadas pelos profissionais de saúde responsáveis pela assistência prénatal, a fim de reduzir a morbidade e mortalidade materna e fetal. Trinta questões clínicas foram priorizadas e deram origem as recomendações. Questões como: nutrição, sintomas da gravidez como náuseas e vômitos, viagens, atividades físicas, entre outras fizeram parte dessas recomendações ${ }^{(8)}$.

Se o cuidado pré-natal é redesenhado para atender as necessidades das mulheres, então é fundamental desenvolver uma compreensão clara de experiências de cuidados pré-natais, assim como a definição das 
mulheres sobre suas próprias necessidades e quais aspectos da assistência pré-natal podem satisfazer essas necessidades $^{(3)}$.

Conhecer as práticas e as reais necessidades de autocuidado exercida pelas gestantes proporciona aos profissionais de saúde reconhecer essas modificações e intervir por meio de orientações específicas durante a consulta de pré-natal ou através de atividades educativas, contribuindo assim para um período gestacional mais saudável, com menos riscos de intercorrências, impactando na saúde do binômio mãefilho.

Embora se trate de tema bastante explorado na literatura, ainda se encontram lacunas ${ }^{(3)}$, visto que orientações adequadas e realização de práticas educativas durante a assistência pré-natal ainda são pouco valorizadas pelos profissionais de saúde. Estudos neste tema podem contribuir com implicações na atuação profissional e na assistência prestada à gestante. Conhecer as práticas de autocuidado realizadas durante a gravidez fornece subsídios para ações educativas direcionadas para suas reais necessidades e qualidade na atenção prestada.

Frente ao exposto, este estudo teve como objetivo verificar as práticas de autocuidado desenvolvidas por gestantes, atendidas pelo serviço de pré-natal de um ambulatório.

\section{METODOLOGIA}

Estudo de caráter descritivo, transversal, com abordagem quantitativa, realizado no serviço de Ginecologia e Obstetrícia do Ambulatório Maria da Glória da Universidade Federal do Triângulo Mineiro AMG/UFTM no município de Uberaba-MG.

A coleta de dados foi realizada de janeiro a fevereiro de 2012. Participaram da pesquisa as gestantes que aguardavam na sala de espera do serviço de Ginecologia e Obstetrícia do referido ambulatório no período do estudo, totalizando 99 participantes.

As entrevistas foram feitas em espaço reservado e individualizado, no período em que as gestantes aguardavam a consulta de pré-natal, sendo realizada durante os dias da semana, de segunda a sexta-feira.

Os critérios de inclusão foram: gestantes maiores de 18 anos atendidas pelo AMG/UFTM.

Foi utilizado um instrumento estruturado, elaborado pelos pesquisadores, baseado em experiências prévias e na literatura científica da área. Compreendia de variáveis sociodemográficas, planejamento da gravidez e realização do pré-natal, questões relacionadas às práticas de autocuidado desenvolvidas pelas gestantes, e orientações recebidas.

Anteriormente ao início da coleta, foi realizado um estudo piloto com 10 gestantes que seriam atendidas no ambulatório a fim de avaliar sua aplicabilidade. As gestantes que realizaram o teste piloto não foram incluídas no estudo.

Os dados coletados foram digitados em planilhas eletrônicas do programa Excelß e, posteriormente, utilizou-se o programa SPSS (Statistical Package for Social Science) versão 17.0 para a análise. Estes dados foram analisados por estatística descritiva, obtendo frequência absoluta e porcentagem para as variáveis categóricas, e frequência média, desvio padrão e mínimo e máximo para variáveis numéricas.

Este estudo é parte integrante do projeto intitulado "Qualidade de Vida e saúde da mulher: Análise Multifocal", aprovado pelo Comitê de Ética em Pesquisa com Seres Humanos da UFTM sob o parecer no 1698.

As entrevistas foram realizadas após a assinatura do Termo de Consentimento Livre e Esclarecido (TCLE), ficando uma via assinada com a pesquisadora e outra com a entrevistada. Após a coleta dos dados, foram oferecidas às participantes informações e orientações baseadas em suas necessidades e dúvidas.

\section{RESULTADOS}

\section{Perfil dos sujeitos}

A Tabela 1 apresenta a caracterização sociodemográfica das gestantes participantes do estudo.

A idade das mulheres participantes do estudo variou de 18 a 38 anos, com média de 25,3 ( $d p=5,1)$ anos. Quanto ao estado conjugal, 48,5\% viviam com o parceiro. A escolaridade formal média das gestantes foi de 8,8 anos de estudo $(\mathrm{dp}=2,5)$ e variou de dois a 14 anos de estudo.

No que se refere à ocupação, 60,6\% não exerciam atividade remunerada. Entre as que possuíam renda individual $(39,4 \%)$, a média salarial foi de 1,3 salários mínimos $(\mathrm{dp}=0,7)$ com o mínimo de um e o máximo de quatro salários mínimos. Sendo que duas gestantes não souberam informar. A renda familiar média mensal das gestantes foi $2,4(\mathrm{dp}=1,6)$ salários mínimos, variando de um a 10 salários. Nesta, 13 mulheres não informaram esse dado. 
Tabela 1: Distribuição das gestantes segundo as variáveis socioeconômicas e demográficas. AMG/UFTM. Uberaba, MG, Brasil, 2012.

\begin{tabular}{|c|c|c|}
\hline Variáveis & No & $\%$ \\
\hline \multicolumn{3}{|l|}{ Grupo etário (anos) } \\
\hline$<20$ & 13 & 13,1 \\
\hline 20 a 24 & 38 & 38,4 \\
\hline 25 a 29 & 23 & 23,2 \\
\hline 30 a 34 & 21 & 21,2 \\
\hline 35 ou mais & 4 & 4 \\
\hline \multicolumn{3}{|l|}{ Escolaridade ( anos de estudo) } \\
\hline Até 8 anos & 39 & 39,4 \\
\hline 9 a 11 anos & 46 & 46,5 \\
\hline 12 ou mais & 14 & 14,1 \\
\hline \multicolumn{3}{|l|}{ Situação conjugal } \\
\hline Casada & 36 & 36,4 \\
\hline Vive junto & 48 & 48,5 \\
\hline Solteira & 12 & 12,1 \\
\hline Separada & 3 & 3 \\
\hline \multicolumn{3}{|l|}{ Ocupação } \\
\hline Do lar & 60 & 60,6 \\
\hline Ocupação remunerada & 39 & 39,4 \\
\hline \multicolumn{3}{|l|}{ Renda individual* (em Salário Minímo) } \\
\hline $1|-| 2$ & 34 & 91,9 \\
\hline $2|-| 4$ & 3 & 8,1 \\
\hline \multicolumn{3}{|l|}{ Renda familiar** (em Salário Minímo) } \\
\hline $1|-| 2$ & 55 & 63,2 \\
\hline $2|-| 4$ & 24 & 27,6 \\
\hline $4|-| 8$ & 7 & 8 \\
\hline Mais de 8 & 1 & 1,1 \\
\hline
\end{tabular}

Quanto ao número de gestações, as participantes apresentaram média de $2,3(\mathrm{dp}=1,5)$, mínimo de um e o máximo de oito. Quando questionadas quanto ao desejo e planejamento da gestação, $75(75,8 \%)$ responderam ter desejado e 54 (54,5\%) delas não.

Quanto à realização ou não do pré-natal todas as 99 (100\%) gestantes relataram estar em acompanhamento, sendo que a média do número de consultas realizadas até 0 momento da entrevista foi de $5,6 \quad(d p=2,7)$, mínimo de uma e o máximo de 15 consultas. Ressalta-se que uma das entrevistadas não soube informar o número de consultas realizadas. Ao serem questionadas quanto ao profissional que realizava as consultas, $71(71,7 \%)$ responderam ser o médico o responsável por seu acompanhamento gestacional, seis $(6,2 \%)$ referiram o enfermeiro e $19(19,8 \%)$ não souberam informar.

\section{Práticas de autocuidado}

Com relação às práticas de autocuidado desenvolvidas pelas gestantes (Tabela 2 ), os resultados evidenciaram que as participantes do estudo apresentaram importante preocupação com relação à alimentação saudável, citando o uso desses alimentos durante a gestação. No interrogatório sobre alimentação a maioria das gestantes, $75,8 \%$ definiu seu apetite como bom. Sendo que a maioria consumia frutas $(58,6 \%)$, leite e derivados $(70,7 \%)$, verduras e legumes $(78,8 \%)$, carboidratos $(96 \%)$ e proteínas $(78,8 \%)$ diariamente. Com relação à hidratação a maioria das gestantes $(70,7 \%)$ alegou consumir mais de um litro (referente a quatro copos) de água por dia.

No que se refere ao consumo de substâncias tóxicas, apontou-se que das entrevistadas, $13,1 \%$ eram tabagistas. Nenhuma relatou o uso de drogas durante a gestação. Em relação ao consumo de bebidas alcoólicas, esse dado foi relatado por $12,1 \%$ gestantes.

Em relação à higiene pessoal $99 \%$ das entrevistadas tomavam banho diariamente e realizavam a lavagem dos cabelos semanalmente. Todas as entrevistadas disseram escovar os dentes diariamente. Porém, a maioria delas $(67,7 \%)$ não fez acompanhamento dentário durante a gestação. Com relação à higiene íntima todas responderam realizá-la diariamente. No quesito vestuário, a maioria das gestantes tem feito uso de roupas confortáveis, passaram a usar sapatos com salto mais baixo e não usavam meias elásticas, no momento.

A maioria $(72,7 \%)$ das gestantes não fazia nenhum tipo de atividade física, dentre as que faziam o tipo de exercício predominante foi a caminhada. Com relação ao padrão de sono e repouso, $49,5 \%$ delas admitiram dormir bem. 
Tabela 2: Distribuição das gestantes segundo as práticas de autocuidado. AMG/UFTM. Uberaba, MG, Brasil, 2012.

\begin{tabular}{|c|c|c|}
\hline Variáveis & No & $\%$ \\
\hline \multicolumn{3}{|l|}{ Alimentação } \\
\hline Bom apetite & 75 & 75,8 \\
\hline Médio apetite & 15 & 15,2 \\
\hline Pouco apetite & 9 & 9,1 \\
\hline \multicolumn{3}{|l|}{ Alimentos maisconsumidos durante o dia } \\
\hline Frutas & 58 & 58,6 \\
\hline Derivados do leite & 70 & 70,7 \\
\hline Verduras/legumes & 78 & 78,8 \\
\hline Carboidratos & 95 & 96 \\
\hline Proteínas & 78 & 78,8 \\
\hline Doces & 43 & 43,4 \\
\hline \multicolumn{3}{|l|}{ Hidratação (ao dia) } \\
\hline Menos de 1 litro & 14 & 14,1 \\
\hline 1 litro & 15 & 15,2 \\
\hline Mais de 1 litro & 70 & 70,7 \\
\hline \multicolumn{3}{|l|}{ Tabagistas } \\
\hline Sim & 13 & 13,1 \\
\hline Não & 86 & 86,9 \\
\hline \multicolumn{3}{|l|}{ Uso de drogas } \\
\hline Não & 99 & 100 \\
\hline \multicolumn{3}{|l|}{ Uso de bebidas alcoólicas } \\
\hline Sim & 12 & 12,1 \\
\hline Não & 87 & 87,9 \\
\hline \multicolumn{3}{|l|}{$\begin{array}{l}\text { Higiene Pessoal } \\
\text { Banho diário }\end{array}$} \\
\hline Sim & 98 & 99 \\
\hline Não & 1 & 1 \\
\hline \multicolumn{3}{|l|}{ Lavagem da cabeça } \\
\hline Sim & 98 & 99 \\
\hline Não & 1 & 1 \\
\hline \multicolumn{3}{|l|}{ Higiene Bucal } \\
\hline Sim & 99 & 100 \\
\hline \multicolumn{3}{|l|}{ Consulta ao dentista } \\
\hline Sim & 32 & 32,3 \\
\hline Não & 67 & 67,7 \\
\hline \multicolumn{3}{|l|}{ Higiene Íntima } \\
\hline Sim & 99 & 100 \\
\hline \multicolumn{3}{|l|}{ Vestuário } \\
\hline Roupas confortáveis & 93 & 93,9 \\
\hline Sapatos de salto baixo & 95 & 96 \\
\hline Meias elásticas & 3 & 3 \\
\hline \multicolumn{3}{|l|}{ Prática de atividade física } \\
\hline $\operatorname{Sim}$ & 27 & 27,3 \\
\hline Não & 72 & 72,3 \\
\hline \multicolumn{3}{|l|}{ Sono e repouso } \\
\hline Dorme bem & 49 & 49,5 \\
\hline Dorme pouco & 26 & 26,3 \\
\hline Tem insônia & 24 & 24,2 \\
\hline
\end{tabular}

Outras práticas de autocuidado podem ser visualizadas na Tabela 3.

Das 99 entrevistadas, 59,6\% responderam não ter problemas com relação à constipação intestinal. Quanto ao padrão miccional $89,9 \%$ das gestantes relataram um aumento deste, sendo que $94,9 \%$ responderam não ter dor durante a micção.

Quando questionadas sobre atividade sexual, 66 $(66,7 \%)$ gestantes responderam manter relações sexuais durante a gestação. Destas, 38 (38,4\%) não relataram desconforto durante o ato. Das $33(33,3 \%)$ gestantes que não estavam mantendo relações no período, 12 $(36,4 \%)$ relataram como motivo a dor durante a relação sexual. A grande maioria das gestantes $(75,8 \%)$ não apresentava corrimento vaginal durante o período gestacional. 
Tabela 3: Distribuição das gestantes segundo as práticas de autocuidado. AMG/UFTM. Uberaba, MG, Brasil, 2012.

\begin{tabular}{|c|c|c|}
\hline Variáveis & No & $\%$ \\
\hline \multicolumn{3}{|l|}{ Constipação intestinal } \\
\hline Sim & 59 & 59,6 \\
\hline Não & 40 & 40,4 \\
\hline \multicolumn{3}{|l|}{ Aumento do padrão miccional } \\
\hline Sim & 89 & 89,9 \\
\hline Não & 10 & 10,1 \\
\hline \multicolumn{3}{|l|}{ Dor ao urinar } \\
\hline Sim & 5 & 5,1 \\
\hline Não & 94 & 94,9 \\
\hline \multicolumn{3}{|l|}{ Atividade sexual } \\
\hline Sim & 66 & 66,7 \\
\hline Não & 33 & 33,3 \\
\hline \multicolumn{3}{|l|}{ Corrimento vaginal } \\
\hline Sim & 24 & 24,2 \\
\hline Não & 75 & 75,8 \\
\hline \multicolumn{3}{|l|}{ Cuidados com as mamas } \\
\hline Uso de sutiã firme de sustentação & 72 & 72,7 \\
\hline Banho de sol nas mamas & 21 & 21,2 \\
\hline Uso de cremes ou óleos na aréola e mamilos & 39 & 39,4 \\
\hline \multicolumn{3}{|l|}{ Trabalho durante a gestação } \\
\hline Sim & 21 & 21,2 \\
\hline Não & 78 & 78,8 \\
\hline \multicolumn{3}{|l|}{ Períodos de repouso } \\
\hline Sim & 14 & 66,7 \\
\hline Não & 7 & 33,3 \\
\hline \multicolumn{3}{|l|}{ Dificuldades no trabalho } \\
\hline $\operatorname{Sim}$ & 11 & 52,4 \\
\hline Não & 10 & 47,6 \\
\hline \multicolumn{3}{|l|}{ Atividade de lazer } \\
\hline $\operatorname{Sim}$ & 58 & 58,6 \\
\hline Não & 41 & 41,4 \\
\hline \multicolumn{3}{|l|}{ Cuidado com estrias } \\
\hline Sim & 55 & 55,6 \\
\hline Não & 44 & 44,4 \\
\hline \multicolumn{3}{|l|}{ Tintura dos cabelos } \\
\hline Sim & 18 & 18,2 \\
\hline Não & 81 & 81,8 \\
\hline \multicolumn{3}{|l|}{ Uso de protetor solar } \\
\hline Sim & 22 & 22,2 \\
\hline Não & 77 & 77,8 \\
\hline \multicolumn{3}{|l|}{ Imunização } \\
\hline Sim & 85 & 85,9 \\
\hline Não & 14 & 14,1 \\
\hline
\end{tabular}

No que se refere ao cuidado com as mamas durante a gestação, a maioria referiu uso do sutiã de sustentação firme, de cremes ou óleos na região da aréola e mamilos, com frequência e a não realização do banho de sol nas mamas.

Das 99 entrevistas, $21 \quad(21,2 \%)$ estavam trabalhando durante a gestação, entre elas, a maioria relatou ter períodos de repouso durante a jornada de trabalho, e ter dificuldades durante sua execução. Ressalta-se que $58,6 \%$ das gestantes referiram realizar atividades de lazer durante o período gestacional.

Alguns cuidados específicos foram descritos pelas gestantes. Em relação as estrias, a maioria das entrevistadas manifestou cuidado para evitá-las, sendo que esse cuidado tem sido feito com o uso de cremes e óleos.

A maioria não realizou tintura nos cabelos durante o período gestacional. E em relação ao uso diário de protetor solar, a grande maioria referiu não usá-lo. A imunização contra o tétano foi realizada por $85,9 \%$ das gestantes.

Com relação às queixas durante a gestação, as que tiveram maior percentual foram: fadiga, lombalgia, pirose e polaciúria como pode ser evidenciado na Tabela 4. 
Tabela 4: Distribuição das gestantes segundo as queixas apresentadas durante a gestação. AMG/UFTM. Uberaba, MG, Brasil, 2012.

\begin{tabular}{ccc}
\hline Variáveis & $\mathbf{N o}^{\mathbf{0}}$ & $\mathbf{\%}$ \\
\hline Fadiga & 80 & 80,8 \\
Lombalgia & 77 & 77,8 \\
Pirose & 72 & 72,7 \\
Polaciúria & 71 & 71,7 \\
Sensibilidades nas mamas & 65 & 65,7 \\
Falta de ar & 56 & 56,6 \\
Edema & 55 & 55,6 \\
Câimbras & 49 & 49,5 \\
Contrações & 41 & 41,4 \\
Flatulências & 36 & 36,4 \\
Náuseas e vômitos & 34 & 34,3 \\
Leucorreia & 18 & 18,2 \\
Hemorróidas & 9 & 9,1 \\
\hline
\end{tabular}

\section{Orientações recebidas}

A maioria das entrevistadas $(68,7 \%)$ afirmou ter recebido orientações sobre o autocuidado na gestação, e ao serem questionadas sobre qual profissional forneceu a orientação, a resposta foi: médico (38,2\%), enfermeiro $(27,9 \%)$ e outra pessoa que não era um profissional de saúde $(33,9 \%)$.

\section{DISCUSSÃO}

De acordo com os resultados descritos, a maioria das gestantes tem média de idade de 25,3 anos, estão em união estável, com escolaridade de 8,8 anos de estudo, não possuíam trabalho remunerado, não planejaram a gestação e tinham realizado uma média de cinco consultas até o momento da entrevista.

Em estudo desenvolvido com 795 puérperas que realizaram pré-natal em Unidade Básica de Saúde /UBS, no município de Santa Maria/RS, os autores encontraram dados próximos ao desta pesquisa, a idade média das entrevistadas foi de 25,2 anos, $86 \%$ tinham companheiro, com média de 8,4 anos de estudo, 59\% não planejaram a gestação e o número médio de consultas foi de $6,3^{(9)}$. Outro estudo realizado em uma maternidade em São Paulo os autores destacaram que $59,8 \%$ das entrevistadas não tinham trabalho remunerado ${ }^{(10)}$.

No que tange às práticas de autocuidado apresentado pelas participantes, estudo realizado com gestantes em quatro UBS da zona Leste de São Paulo/SP apontou que a maioria tomava banho de uma a duas vezes ao dia, 32,3\% faziam a higiene dos cabelos duas vezes por semana, $55,6 \%$ ingeriam de um a dois litros de líquidos diariamente, $72,6 \%$ não faziam uso do protetor solar, $86,3 \%$ não realizavam qualquer tipo de atividade física(11). Esses dados estão próximos aos encontrados no presente estudo. Destaca-se a pouca importância dada pelas gestantes em relação ao uso diário de protetor solar e a realização de atividade física. Essa pouca importância pode ser compreendida dado ao fato dessas gestantes não terem informações adequadas sobre a sua importância de uso e realização neste período.

Quanto ao uso de substâncias tóxicas à gestação, a maioria das gestantes relatou não ter o hábito de fumar e nem de ingerir bebidas alcoólicas durante a gestação. Estudo realizado com 2.557 puérperas em um hospital universitário da Universidade Federal do Rio Grande apontou que 1.846 não fumaram na gestação e 2.461 não fizeram uso de bebidas alcoólicas, condizendo com os dados encontrados na pesquisa apresentada ${ }^{(12)}$.

Quanto à saúde bucal todas as entrevistadas relataram escovar os dentes diariamente, porém durante - período gestacional a maioria não fez acompanhamento com dentista. Houve mudança no vestuário, com uso de roupas mais confortáveis e uma alimentação variada e equilibrada.

Estudo realizado com gestantes adolescentes em Bogotá na Colômbia apontou grande interesse das entrevistadas em cuidar dos dentes, frequentando o dentista regularmente, com aumento do número de escovações e do uso do fio dental, isso devido a conscientização de que esta fase pode ocorrer alterações dentárias $^{(13)}$. As grávidas correm maior risco de apresentar a cárie dentária por diversas razões, incluindo o aumento da acidez na cavidade bucal, a elevação da frequência da ingestão de alimentos e por causa da pouca atenção que elas dão para a saúde bucal ${ }^{(2)}$.

Ainda, no estudo realizado em Bogotá, os autores revelaram que em relação a alimentação houve o predomínio do consumo de leite e derivados, frutas e carnes. Observou-se também maior atenção ao vestuário, evitando roupas justas e maior preferência 
àquelas que proporcionem conforto a gestante ${ }^{(13)}$. Esses dados vão ao encontro da presente pesquisa, visto que as gestantes referiram alimentação saudável. Os cuidados com vestuários adequados também foram citados, proporcionado assim conforto e comodidade no dia a dia.

Quando questionadas sobre o cuidado com as mamas durante a gestação a maioria referiu executá-los usando sutiã de sustentação e usando cremes e óleos na região da aréola e mamilos, porém o banho de sol foi pouco realizado. Segundo o Ministério da Saúde, as mamas devem ser preparadas para o aleitamento materno ainda na gestação. Para que isso ocorra, o profissional de saúde deve avaliar as mamas da gestante em todas as consultas de pré-natal, orientar quanto ao uso de sutiã durante a gestação e expor as mamas ao banho de sol por 15 minutos no período de até as 10 horas da manhã e após as 16 horas ou banhos de luz com lâmpadas de 40 watts, a cerca de um palmo de distância e evitar o uso de cremes, sabões ou pomadas na região dos mamilos ${ }^{(2)}$.

Com esses dados pôde-se identificar a prática errada no cuidado com as mamas, por meio do uso de óleos e cremes na região da aréola e mamilos. Isso pode caracterizar a falta de orientação durante o pré-natal. Orientação esta que poderia ser realizada na consulta, na sala de espera por meio das atividades educativas e também nas visitas domiciliares realizadas pelas equipes de Estratégia de Saúde da Família.

Em outro estudo com 30 puérperas de um Hospital da rede pública de São Paulo, 30\% afirmaram não ter recebido nenhuma orientação durante o pré-natal sobre os cuidados com as mamas durante a gestação. $O$ cuidado com as mamas durante a gestação interfere diretamente no ato de amamentar, já que se a mama estiver preparada para este ato, o aleitamento materno se torna mais fácil e a possibilidade de boa aceitação aumenta $^{(14)}$.

A presente pesquisa apontou que a maioria das entrevistadas não apresentou sintomas de constipação intestinal durante a gestação, porém quanto ao padrão miccional foi percebido um aumento da frequência de micções. Alterações no padrão eliminatório das gestantes são comuns. A constipação intestinal é causada pelo aumento da taxa de hormônios esteroides que diminuem a motilidade da musculatura intestinal e também pelo deslocamento que o útero aumentado causa nas alças intestinais $^{(15)}$. É recomendado orientar a gestante a ter uma dieta rica em fibras e aumentar a ingesta hídrica para minimizar esse incômodo. O aumento do número de micções é comum no início e no final da gestação (devido ao aumento do útero e à compressão da bexiga). Mesmo sendo incômodo o aumento do número de micções, é de extrema importância incentivar a ingestão hídrica adequada $^{(2)}$.

O estudo evidenciou que dentre as gestantes entrevistadas a maioria mantinha relações sexuais durante a gravidez, já as que não as realizam apontaram a dor como impedimento ao ato. Outras queixas foram relatadas com maior frequência: fadiga, lombalgia, pirose e polaciúria.

Um grupo de 12 gestantes entrevistadas em uma unidade básica de saúde de São Paulo relatou diversas alterações durante a gestação, sendo consideradas desconfortantes. Foi citado com maior importância, o aumento da sensibilidade das mamas, enjôos, vômitos, pirose e edema. Algumas relataram ainda perda da libido, ocorrência de sangramento vaginal e dor ao praticar o ato sexual ${ }^{(16)}$. As queixas apresentadas pelas gestantes são decorrentes de processos fisiológicos e anatômicos decorrentes desse período, mas que podem ser minimizados por meio de orientações específicas para cada queixa.

Apesar de a pesquisa apresentar uma pequena porcentagem $(21,2 \%)$ de gestantes que estavam trabalhando no período, uma gestação saudável e sem intercorrências não contra indica a mulher a manter seu emprego, desde que este seja em um ambiente seguro e que não exponha a gestante a riscos contra sua saúde. Além disso, devem ser respeitados os momentos de descanso durante a jornada de trabalho ${ }^{(15)}$.

Cada gestante tem uma especificidade no trabalho realizado, por isso orientações específicas devem ser valorizadas, a fim de proporcionar horas de trabalho mais saudáveis, sem riscos de intercorrências.

As gestantes entrevistadas relataram não ter feito uso de tinturas no cabelo durante o período gestacional. Recomenda-se que esse ato não seja realizado até o terceiro mês de gestação, após esse período não há evidencias que proíbam o uso, porém é importante evitar o uso de tinturas com presença de metais pesados e amônia durante todo o processo gestacional ${ }^{(15)}$.

O aparecimento de estrias é uma das modificações da pele mais preocupantes para a mulher na fase gestacional, sendo que no presente estudo $55,6 \%$ das entrevistadas estavam cuidando da pele para evitá-las. Em uma pesquisa com 124 gestantes da unidade básica de saúde regional da Penha em São Paulo, os autores evidenciaram que $91,2 \%$ das participantes apresentavam alterações na pele durante a gestação, 
sendo que $17,1 \%$ perceberam o surgimento de estrias na região do abdome e glúteo ${ }^{(17)}$.

As estrias são resultantes da distensão dos tecidos e não existe método eficaz de prevenção. Ainda que controversas, podem ser utilizadas massagens locais, com substâncias oleosas ou cremes, na tentativa de preveni-las $^{(2)}$.

A pesquisa confirmou que a imunização contra o tétano foi realizada por $85,9 \%$ das gestantes entrevistadas. A vacina é indicada para a proteção da gestante contra o tétano acidental e a prevenção do tétano neonatal ${ }^{(2)}$.

Quanto às orientações sobre autocuidado recebidas por profissional de saúde, o estudo demonstrou que a maioria das gestantes tinha recebido. Em uma revisão integrativa, os autores identificaram que quando o cuidado pré-natal é mais abrangente, com orientações, as mulheres em geral respondem mais positivamente ao cuidado $^{(3)}$.

A ausência ou ineficiência de orientações por parte dos profissionais, levam as mulheres a buscarem informações em outras fontes como família, amigos, televisão e revistas ${ }^{(18)}$. Orientações estas muitas vezes errôneas, podendo influenciar nas práticas de autocuidado e prejudicar a qualidade de vida da gestante

As orientações durante o pré-natal são capazes de promover educação em saúde em diversos âmbitos de abordagem, sejam essas em grupos ou individualmente. A interação conseguida entre profissionais de saúde e gestantes gera troca de experiências, fortalecimento de vínculo, aumento de conhecimento e resolução de dúvidas comuns ao período gestacional ${ }^{(9)}$.

\section{CONCLUSÃO}

O estudo possibilitou verificar como as gestantes têm cuidado de sua saúde, e algumas atitudes realizadas por elas. Dados que permite evidenciar que muitas gestantes não apresentam orientações adequadas sobre sintomatologias presentes neste período. Realizando ações inadequadas pela falta de conhecimento. Diante dos achados, percebemos que ainda há uma lacuna na atenção oferecida pelos profissionais de saúde responsáveis pelo atendimento pré-natal, visto que $31,3 \%$ das participantes referiram não ter recebido orientações neste período. O presente estudo reforçou dados da literatura que evidenciaram a importância das práticas educativas no pré-natal.

As orientações de autocuidado na gestação deveriam ser iniciadas durante as primeiras consultas de pré-natal e até mesmo por meio de grupos educativos e sala de espera. A visita domiciliar realizada pelas equipes da Estratégia Saúde da Família, também é essencial neste período.

Acredita-se que os profissionais de saúde e os serviços de saúde têm papel fundamental neste processo, que envolve a saúde do binômio mãe-filho. A criação de programas educativos e a reorganização do atendimento dos serviços de saúde são estratégias que podem contribuir para efetivar a qualidade da assistência prestada no pré-natal.

É importante a qualificação e estímulo dos profissionais de saúde para lidar com esse período de maneira adequada, principalmente os enfermeiros, que são profissionais capacitados para reconhecer as ações de autocuidado e agir conforme as necessidades da gestante.

Espera-se que com este estudo haja uma sensibilização dos profissionais de saúde e estudantes da área. Embora a pesquisa tenha como limitação ser um recorte transversal, os achados apontados aqui corroboram com as informações de outros estudos, podendo apontar para similaridades nos aspectos relacionados as práticas de autocuidado entre gestantes.

\section{REFERÊNCIAS}

1. Magalhães RS, Costa MS, Matsue RY, Sousa GS, Catrib AMF, Vieira LJES. Cartografia do cuidado na saúde da gestante. Cien Saude Colet [Internet]. 2012 [acesso em: $31 \mathrm{dez}$ 2014];17(3):635-42. Disponível em: http://dx.doi.org/10.1590/S1413-81232012000300009. 2. Ministério da Saúde. Atenção ao pré-natal de baixo risco [Internet]. Brasília: Ministério da Saúde; 2012 [acesso em: 31 dez 2014]. Disponível em:

http://bvsms.saude.gov.br/bvs/publicacoes/cadernos_atencao_b asica_32_prenatal.pdf.

3. Novick G. Women's Experience of Prenatal Care: An Integrative Review. J Midwifery Womens Health [Internet]. 2009 [acesso em: $31 \mathrm{dez} 2014] ; 54(3): 226-37$. Disponível em: http://dx.doi.org/10.1016/j.jmwh.2009.02.003.

\footnotetext{
4. Ramazani N, Zareban I, Ahmadi R, ZadSirjan S, Daryaeian M. Effect of Anticipatory Guidance Presentation Methods on the Knowledge and Attitude of Pregnant Women Relative to Maternal, Infant and Toddler's Oral Health Care. J Dent (Tehran) [Internet]. 2014 [acesso em: $31 \mathrm{dez} 2014$ ];11(1):22-9. Disponível em:

http://jdt.tums.ac.ir/index.php/jdt/article/view/1025.

5. Ministério da Saúde. Pré-natal e Puerpério: atenção qualificada e humanizada - manual técnico [Internet]. Brasília: Ministério da Saúde; 2005 [acesso em: 31 dez 2014]. Disponível em: http://bvsms.saude.gov.br/bvs/publicacoes/manual_pre_natal_p uerperio_3ed.pdf.

6. Pessôa IN, Menezes ED, Ferreira TF, Dotto LMG, Bessa LF. Percepção de puérperas sobre assistência de enfermagem na gravidez. Ciênc. cuid. Saúde [Internet]. 2009 [acesso em: 31
} 
dez 2014];8(2):236-41. Disponível em:

http://dx.doi.org/10.4025/cienccuidsaude.v8i2.8204.

7. Souza VB, Roecker S, Marcon SS. Ações educativas durante a assistência pré-natal: percepção de gestantes atendidas na rede básica de Maringá-PR. Rev. Eletr. Enf. [Internet]. 2011 [acesso em: $31 \mathrm{dez} 2014] ; 13(2): 199-210$. Disponível em:

http://dx.doi.org/10.5216/ree.v13i2.10162.

8. Amaya-Guío J, Díaz-Cruz LA, Cardona-Ospina A, RodríguezMerchán DM, Osorio-Sánchez D, Barrera-Barinas A. Guía de Práctica Clínica para la prevención y detección temprana de las alteraciones del embarazo. Rev Colomb Obstet Ginecol

[Internet]. 2013 [acesso em: $31 \mathrm{dez} 2014$ ];64(3):245-88.

Disponível em:

http://www.fecolsog.org/userfiles/file/revista/Revista_Vol64No3_ Julio_Septiembre_2013/v64n3a05.pdf.

9. Anversa ETR, Bastos GAN, Nunes LN, Dal Pizzol TS. Qualidade do processo da assistência pré-natal: unidades básicas de saúde e unidades de Estratégia Saúde da Família em município no Sul do Brasil. Cad Saude Publica [Internet]. 2012 [acesso em: 31 dez 2014];28(4):789-800. Disponível em: http://dx.doi.org/10.1590/S0102-311X2012000400018.

10. Corrêa CRH, Bonadio IC, Tsunechiro MA. Normative prenatal evaluation at a philanthropic maternity hospital in São Paulo. Rev Esc Enferm USP [Internet]. 2011 [acesso em: $31 \mathrm{dez}$ 2014];45(6):1293-300. Disponível em:

http://dx.doi.org/10.1590/S0080-62342011000600003. 11. Urasaki MBM. Cuidados com a pele adotados por gestantes assistidas em serviços públicos de saúde. Acta paul. enferm. [Internet]. 2011 [acesso em: $31 \mathrm{dez} 2014$ ];24(1):67-73. Disponível em: http://dx.doi.org/10.1590/S010321002011000100010.

12. Prietsch SOM, González-Chica DA, Cesar JA, Mendoza-Sassi RA. Gravidez não planejada no extremo Sul do Brasil: prevalência e fatores associados. Cad Saude Publica [Internet]. 2011 [acesso em: 31 dez 2014];27(10):1906-16. Disponível em: http://dx.doi.org/10.1590/S0102-311X2011001000004. 13. Rativa Martínez N, Ruiz de Cárdenas $\mathrm{CH}$. Si protegemos la vida y la salud durante la gestación, construimos para los dos un futuro saludable. Av. Enferm [Internet]. 2009 [acesso em: 31 dez 2014];XXVII(1):30-7. Disponível em:

http://www.enfermeria.unal.edu.co/revista/articulos/xxvii1_4.pdf

14. Souza MJN, Barnabé AS, Oliveira RS, Ferraz RRN. A

importancia da orientação à gestante sobre amamentação: fator para diminuicão dos processos dolorosos mamários. ConScientiae Saúde [Internet]. 2009 [acesso em: 31 dez 2014];8(2):245-9. Disponível em:

http://www.uninove.br/PDFs/Publicacoes/conscientiae_saude/csa ude_v8n2/cnsv8n2_3k1475.pdf.

15. Zampieri MFM, Garcia ORZ, Boehs AE, Verdi M. Enfermagem na atenção primária à saúde da mulher. Florianópolis: USFC; 2007.

16. Araújo NM, Salim NR, Gualda DMR, Silva LCFP. Body and sexuality during pregnancy. Rev Esc Enferm USP [Internet]. 2012 [acesso em: $31 \mathrm{dez} 2014$ ];46(3):552-8. Disponível em: http://dx.doi.org/10.1590/S0080-62342012000300004.

17. Urasaki MBM. Alterações fisiológicas da pele percebidas por gestantes assistidas em serviços públicos de saúde. Acta paul. enferm. [Internet]. 2010 [acesso em: $31 \mathrm{dez} 2014$ ];23(4):51925. Disponível em: http://dx.doi.org/10.1590/S0103-

21002010000400012 .

18. Guerreiro EM, Rodrigues DP, Queiroz ABA, Ferreira MA, Rodrigues IR, Melo LPT. Representações sociais de puérperas sobre $o$ atendimento pré-natal na atenção primária de saúde. Rev. RENE. [Internet]. 2013 [acesso em: $31 \mathrm{dez}$ 2014];14(5):951-9. Disponível em:

http://www.revistarene.ufc.br/revista/index.php/revista/article/vi ew/1316.

Artigo recebido em 20/12/2012.

Aprovado para publicação em 23/04/14.

Artigo publicado em 31/12/2014. 